\title{
Visualization Teaching of Deformation Monitoring and Data Processing based on MATLAB
}

\author{
https://doi.org/10.3991/ijet.v14i02.9983 \\ Ning Gao \\ Henan University of Urban Construction, Pingdingshan, China \\ gaoninghaoyun@ $163 . \mathrm{com}$
}

\begin{abstract}
As one of the core professional courses for students majoring in surveying and mapping engineering, deformation monitoring is characterized by strong theories, numerous mathematical formulas, and complicated calculation. Task-driving teaching method in the form of traditional classroom has poor effects due to the one-way learning and strategy. To meet the needs of modern teaching, matrix laboratory (MATLAB) and deformation monitoring teaching are combined in this study. This work first identified the aspects of concepts, principles, and data to visualize boring data and abstract principles through simulation and concepts that are difficult to be understood and establishment of animation models to realize the visualization expression of deformation monitoring data preprocessing, deformation monitoring network adjustment, deformation prediction, and engineering application. A total of 114 students majoring in surveying and mapping engineering in Henan University of Urban Construction during 2014 are selected as test subjects to explore and demonstrate visualization teaching of deformation monitoring. Results show that the visualization teaching of deformation monitoring based on MATLAB enhances the integrity, coherence, and practicability of the course. It also improves students' interest in learning, deepens their understanding on learned knowledge, improves teaching quality, and provides a new way for the teaching mode reform of other courses of the surveying and mapping engineering specialty.
\end{abstract}

Keywords-Deformation monitoring, MATLAB,visualization, data processing

\section{$1 \quad$ Introduction}

With the progress of human society and rapid development of world economy, all kinds of construction engineering have been accelerated in recent years. In this context, various types of surface and underground engineering projects have emerged. These works include construction of high-rise buildings, large-scale water conservancy projects and urban underground transportation network, excavation of river-crossing and sea-crossing tunnels, and mining of various mineral resources. However, various geological disasters have occurred due to the excessive destruction of the environment and unreasonable exploitation and utilization of space. Samples of these disasters are ground subsidence caused by overexploitation of underground 
solid, gas, and liquid resources, building subsidence caused by surface load changes, and local earthquakes and landslides induced by dam storage. The occurrence of these disasters has caused tremendous threats and serious losses to the production, life, and property of human beings all over the world. Practice has proven that the occurrence of these disasters is closely related to deformation. Therefore, deformation has become one of the most important research subjects in surveying, mapping, and other related disciplines in monitoring the deformation of various natural and man-made buildings, conducting further analysis, interpreting data, and providing accurate predictions [1].

Many colleges and universities have added deformation monitoring and data processing to surveying and mapping courses. They list it as a compulsory basic course for students in this major. Deformation monitoring and data processing mainly include deformation monitoring technology and monitoring data analysis and prediction. The mathematical model and data processing of deformation monitoring data are the core contents of the course. This course also involves knowledge of many subjects, such as higher mathematics, linear algebra, probability theory and mathematical statistics, matrix theory, and surveying. Excessive mathematical formula derivations and complex calculations bring great difficulties to the teaching of the course. On the one hand, excessive theoretical formula derivations make students feel monotonous and bored, and the teaching time is not enough to teach many calculation examples. On the other hand, despite the available computer programming ability before learning the course, students cannot easily write largescale computer programs. In addition, excessive time spent in programming inevitably affects the understanding and mastery of the contents of the course, which will be counterproductive to its study [2] [3]. Therefore, most students experience difficulty in learning the course and teachers are challenged in mobilizing students' interest in learning.

To solve these problems, deformation monitoring and data processing are demonstrated via an interactive environment and visualization function of MATLAB platform.

\section{State of Art}

MATLAB is a mathematical software with visual expression and interactive program integration published by Math Works for scientific computing. MATLAB takes a matrix as the basic data structure and vector and scalar as the special matrix. Hence, it has excellent numerical calculation ability and data visualization function. Its operating system mainly includes five parts, MATLAB language system, working environment, graphics processing, data function library, and application program interface. MATLAB data function library includes sine, complex operation, and other simple functions and involves matrix transposition, matrix inversion, matrix operation, and other complex functions that provide basis for data processing. MATLAB also has a powerful drawing function, which can display data in 2D and 3D. In addition, the additional toolbox of MATLAB can perform various complex 
dataset analyses [5][6], such as data regression analysis, curve fitting, grey prediction [7], wavelet transform, back propagation(BP) neural network, and wavelet neural network. Thus, MATLAB is superior in data processing.

The application of MATLAB in complex deformation monitoring and data processing can increase data process speed and ensure accuracy of data processing. For example, Tao Qiuxiang realized the adjustment calculation of GPS network by using MATLAB, which enhances students' interest in studying the specialized course [8]. Li Wei studied the transformation models of different coordinate systems and wrote programs using MATLAB programming language on the software platform to realize the programming of coordinate transformation models of different and similar reference ellipsoids. He also found that the transformation of the coordinate system and fast operation speed can be achieved well via MATLAB [9]. Gao Caiyun optimized the parameters of BP neutral network using MATLAB to realize its deformation prediction, thereby obtaining a good prediction effect [10]. Chen Lin improved the wavelet neural network based on MATLAB and applied it to the horizontal displacement prediction of subway tunnel monitoring[11]. The research results of the scholars show the following shortcomings of deformation monitoring and data processing with MATLAB,

- The research conducted a deformation data process with MATLAB only from a single angle, which lacks integrity.

- The degree of visualization in deformation data processing is not high.

Therefore, by combining MATLAB with deformation monitoring and data processing, the current study first identified the deformation data and basic principles. It aims to visualize boring data and abstract principles using simulation technology and difficult concepts by establishing animation models and to realize the visual expression of four modules, namely, deformation monitoring data preprocessing, deformation monitoring network adjustment, deformation forecasting, and engineering application.

\section{Visualization of Deformation Monitoring and Data Processing based on MATLAB}

\subsection{Construction of the Visualization System for Deformation Monitoring and Data Processing}

Deformation monitoring and data processing mainly include deformation monitoring technology and monitoring data analysis and prediction. Through the study of this course, students should master the methods of monitoring the deformation of various deformation bodies (such as high-rise buildings, dams, bridges, and mines), design of deformation monitoring schemes, adjustment calculation of the deformation monitoring network, and deformation analysis and prediction. The mathematical model and data processing of deformation monitoring data are the core contents of the course that need to be mastered by students. In 
accordance with the recent deformation monitoring teaching and scientific research practice and based on the characteristics and development trend of the discipline, the author constructed a visualization system of deformation monitoring and data processing based on MATLAB, as shown in Fig1.

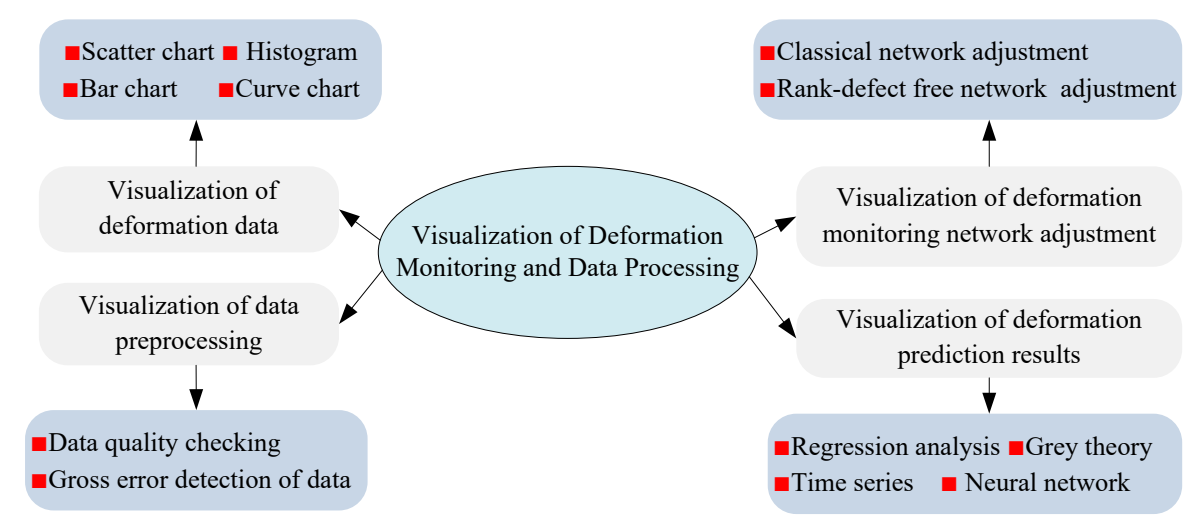

Fig. 1. Visualization teaching system of deformation monitoring and data processing

The system consists of four modules, visualization of deformation data, visualization of data preprocessing, visualization of deformation monitoring network adjustment, and visualization of deformation prediction results.

- The visualization of deformation data includes scatter chart, histogram, bar chart, and curve chart.

- The visualization of data preprocessing includes data quality checking and gross error detection of data.

- The visualization of deformation monitoring network adjustment includes classical network and rank-defect free net adjustments.

- The visualization of deformation prediction results includes regression analysis, grey systems theory, time series, and neural network.

\subsection{Visualization of Deformation Monitoring Data}

The targets of deformation monitoring vary, ranging from the entire earth to a single engineering building. The reasons for deformation are different, resulting in different laws of deformation data. The data obtained from monitoring various deformation bodies contain inherent causes and laws of deformation. Therefore, the monitoring data must be analyzed. MATLAB has powerful 2D and 3D graphics drawing functions. Hence, it can be used to realize the visualization of monitoring data. Data visualization refers to the graphical representation of data. It can vividly describe the characteristics and distribution of data using geometric graphics, such as bar chart, histogram, curve chart, stair chart, and contour map. 

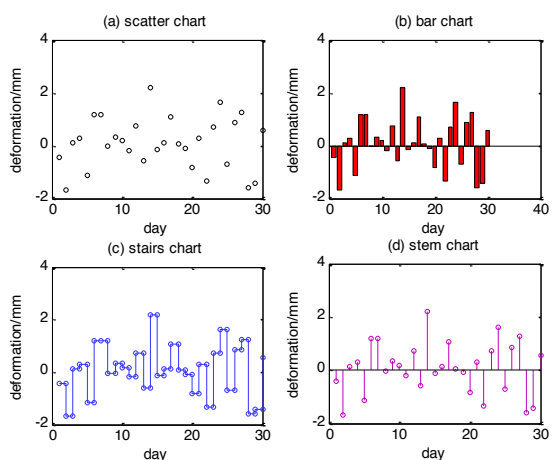

Fig. 2. Visualization of the settlement deformation data of high-rise buildings

In Fig. 2, the settlement and deformation data of a high-rise building are visually displayed by the scatter, bar, stair, and stem charts.

For example, 110 elevation points are collected in a mining subsidence area of a certain mine in China. The 3D topographic map and contour map of the area are generated using MATLAB, as shown in Figs. 3 and 4.

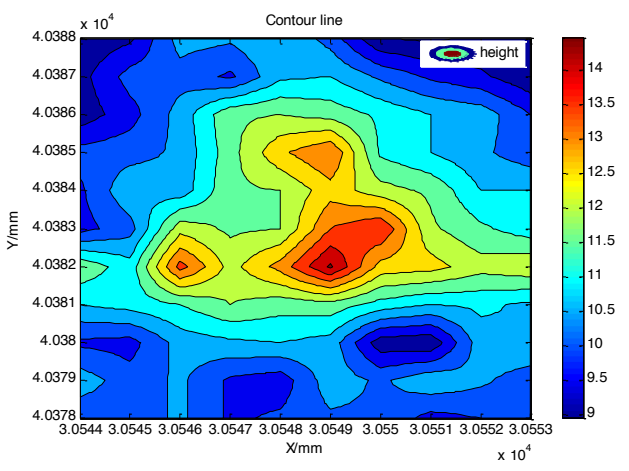

Fig. 3. Contour map of mining subsidence

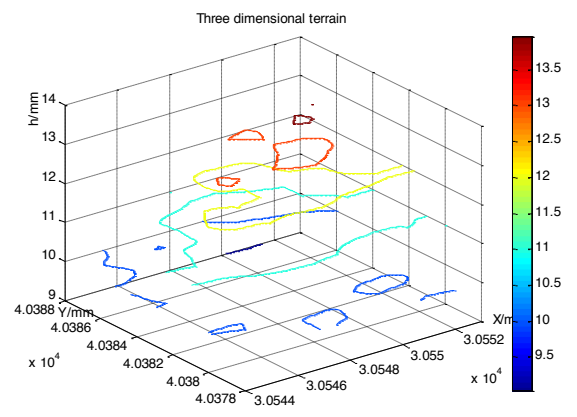

Fig. 4. 3D visualization of mining subsidence topography 


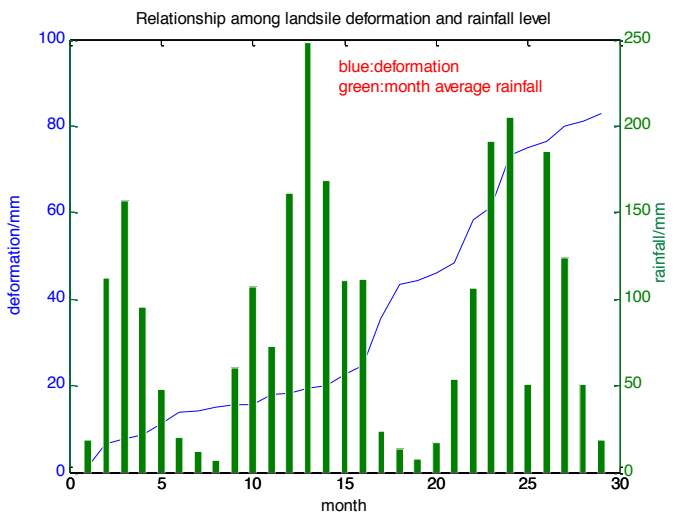

Fig. 5. Relationship between the accumulated displacements of a landslide monitoring point in Three Gorges Reservoir area and rainfall level

In addition, the relationship between environmental impact factors and deformations must be considered when studying special deformation bodies. Taking a landslide in the Three Gorges Reservoir Area of China as an example, the landslide body is mainly the Quaternary-Holocene landslide accumulation layer and the lithology is mainly silty clay. The front of the landslide is adjacent to the Yangtze River, which has been wading into the water. The rheological behavior of the rocksoil body in the landslide is controlled by its own nature, sliding weight, and boundary conditions and strongly influenced by rainfall, water level of the reservoir, and other external induced factors. The relationship between rainfall level and landslide deformation is shown in Fig. 5.

\subsection{Visualization of Deformation Monitoring Data Preprocessing}

Before modeling data adjustment, the data must be preprocessed, mainly including data quality checking and gross error detection of the monitoring data.

- Monitoring data checking: First, the quality of the monitoring data is analyzed, including the accuracy and reliability of the observed values.

- Gross error detection: The gross errors exist in the observed data of any monitoring system. The distribution of gross errors does not obey the orthodox distribution. However, it has great randomness, which has a serious impact on subsequent data processing. Therefore, gross error detection should be performed.

For example, the grey envelope curve method is used to detect the gross errors of deformation monitoring data [12]. A certain deformation is observed for $n$ times of periodic observation. The observation sequence is $x^{(0)}=\left\{x^{(0)}(1), x^{(0)}(2), \cdots x^{(0)}(n)\right\}$. It ranged from small to large. The $P$ th observing point is set as the turning point of the grey envelope. The value of $P$ is as 


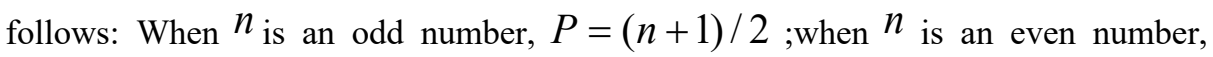
$P=n / 2$

The upper bound of the grey envelope curve is the mean cumulative curve, namely,

$$
x_{\max }=\bar{x} k
$$

The lower bound of the grey envelope curve is

$$
x_{\min }=\left\{\begin{array}{cc}
\bar{x} k-\frac{h \Delta_{\max } k}{p} & (1 \leq k \leq p) \\
\bar{x} k-\frac{h \Delta_{\max }(n-k)}{n-p} & (p<k \leq n)
\end{array}\right.
$$

In Equation (2), $h$ is a constant. After many statistical calculations, $h=3.75$.

If the cumulative value of observations $x^{(1)}(k)$ satisfied

$$
x_{\min } \leq x^{(1)}(k) \leq x_{\max }
$$

it is considered that there is no gross error in the observed data. The detection results are shown in Fig. 6.

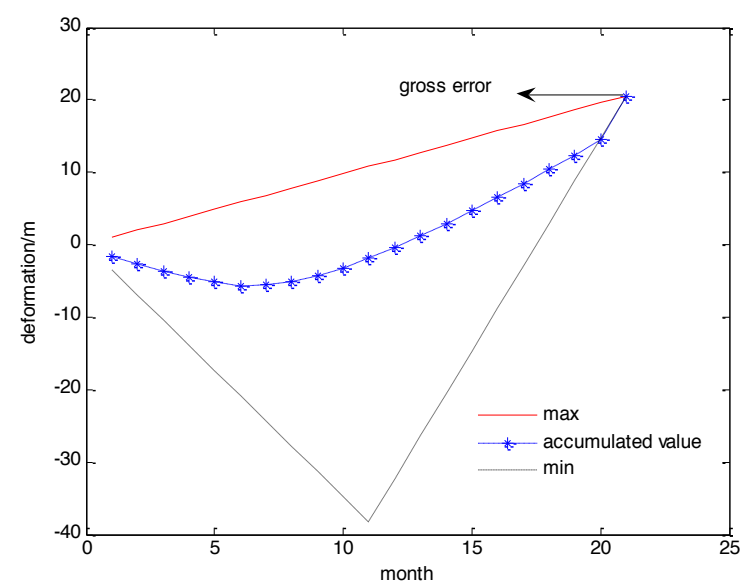

Fig. 6. Grey envelope for gross error detection

\subsection{Visualization of Deformation Monitoring Network Adjustment}

Gross errors can be eliminated from the observed data and materials of the deformation monitoring network after preprocessing. At this time, the data of the 
deformation monitoring network, that is, adjustment calculation, should be processed [13], which mainly includes two parts,

- Classical free net adjustment. The classical free net adjustment can be used whenenough stable points are available around the deformed body as the initial data and database to determine the coordinates of other monitoring points.

- Rank-defect free net adjustment. The rank-defect free net adjustment is applied in the absence ofinitial data around the deformed body due to the rank defect of the coefficient matrix of the normal equation.

To realize the visualization of the adjustment calculation of deformation monitoring network, the author developed the software of deformation data adjustment using MATLAB, as shown in Fig. 7.

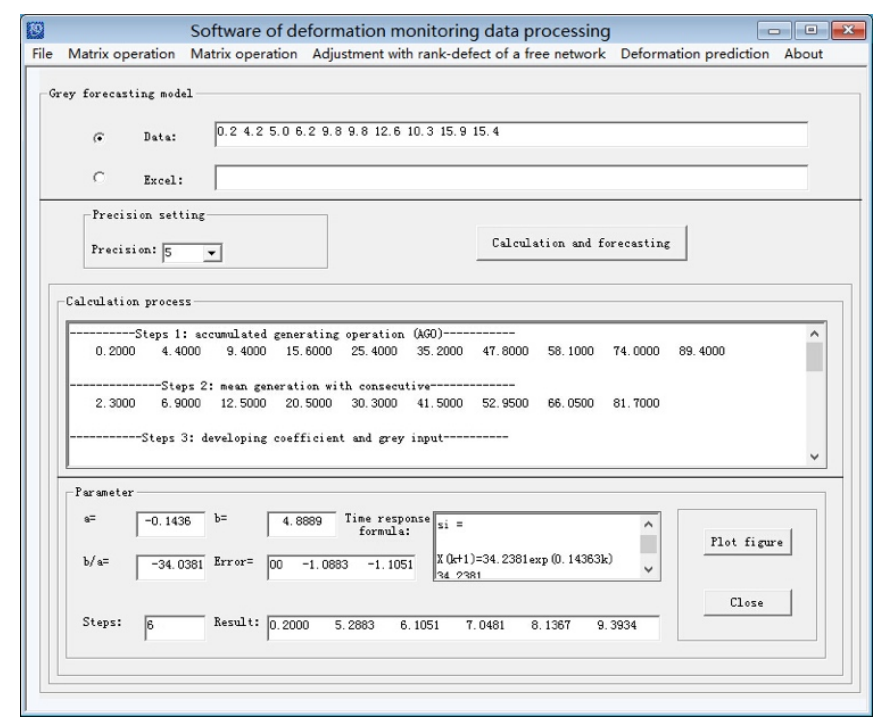

Fig. 7. Deformation adjustment software based on MATLAB

\subsection{Visualization of Deformation Prediction}

The causes for the deformation of the deformed body are very complicated. Generally, such complication is related to many factors, such as physical properties of the deformed body, mechanical properties, geological conditions, and external environment. Thus, the deformation is characterized by uncertainty, intricacy, and complexity, which cause various deformation analysis theories and methods, such as regression analysis, grey system model, and artificial neural network [14][15][16].

The visualization of the prediction method can be realize using MATLAB. A visualization process of the deformation prediction of a dam by BP neural network is shown in Fig. 8. 


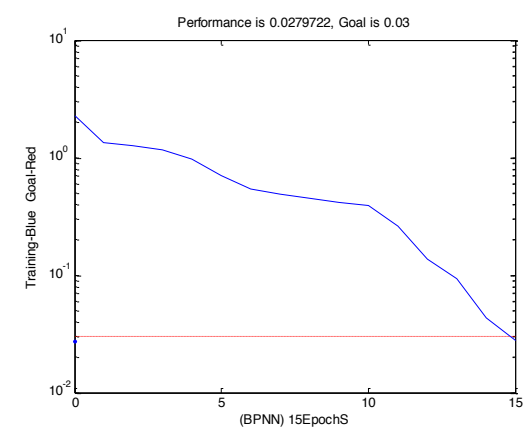

Fig. 8. Visualization of deformation prediction for BP neural network

Effectiveness Analysis of the Implementation of the Visualization Teaching System

To test the visualization teaching effect of deformation monitoring based on MATLAB, it is applied to the teaching of deformation monitoring and data processing for 114 students majoring in surveying and mapping engineering in Henan University of Urban Construction during 2014.

After introducing animation models and MATLAB simulation into the deformation monitoring classroom teaching, students' enthusiasm for learning ran high. An increasing number of students actively asked questions during breaks, and the teaching effect improved significantly, As shown in Table 1. The error ellipse of deformation accuracy evaluation realized by students' independent programming is shown in Fig. 9.

Table 1. Visualization teaching effect analysis

\begin{tabular}{|l|c|c|}
\hline \multicolumn{1}{|c|}{ Teaching Mode } & Traditional Teaching & Visualization Teaching \\
\hline Active questioning rate & $41 \%$ & $90 \%$ \\
\hline Mastery rate of deformation concept and principle & $33 \%$ & $72 \%$ \\
\hline Mastery rate of programming & $27 \%$ & $73 \%$ \\
\hline $\begin{array}{l}\text { Mastery rate of adjustment algorithm of deformation } \\
\text { monitoring network }\end{array}$ & $42 \%$ & $87 \%$ \\
\hline Cognition degree of deformation monitoring tasks & $50 \%$ & $89 \%$ \\
\hline
\end{tabular}

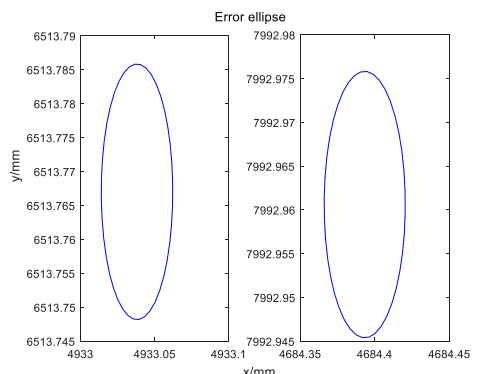

Fig. 9. Error ellipses drawing realized by students programming 
Thus, the visualization teaching system can enable efficient teaching and provides a deep analysis on the instructive and practical theories according to the characteristics of each module[17]. The system can also give a general explanation of other contents. This kind of teaching has its focus on theories, so it can be exerted incisively and vividly. The teaching contents can also be optimized constantly and the quality of practical teaching be improved.

Conclusions

To make up for the deficiency of the traditional teaching mode of deformation monitoring, the visualization technology of MATLAB is applied to the teaching of deformation monitoring to construct the visualization system of deformation monitoring and data processing based on MATLAB. Thus, the study realizes the visualization expression of four modules, namely, deformation monitoring data preprocessing, deformation monitoring network adjustment, deformation prediction, and engineering application. MATLAB is used in the teaching of deformation monitoring for students majoring in surveying and mapping engineering in Henan University of Urban Construction during 2014. We draw the following conclusions,

- Visualization teaching is a new teaching mode that can meet the learning needs of different levels of learners and provide the possibility of teaching students in accordance with their aptitude.

- The visualization teaching system based on MATLAB clarifies the learning goals and tasks of learners. It also improves the visualization and concreteness of complex engineering cases using four modules, namely, deformation monitoring data preprocessing, deformation monitoring network adjustment, deformation prediction, and engineering application, which can be easily learned by students independently.

- The visualization teaching based on MATLAB focuses on the principle of data processing and integrated students' preview in advance, program development, and teachers' classroom demonstration. Thus, it improves students' level in deformation monitoring and data processing engineering application.

At present, the visualization teaching mode of deformation monitoring based on modeling and simulation is still being explored. The method has high requirements on teachers' personal ability and lesson preparation energy. Therefore, identifying how to develop high-quality teaching resources through synergetic division of labor and how to combine it with other teaching modes organically to produce ideal teaching effect are the questions that need further thinking and research.

\section{$4 \quad$ References}

[1] Gao Ning, Gao Caiyun, Exploration of modular teaching of the deformation monitoring and data processing course in the surveying and mapping engineering major, World Transactions on Engineering and Technology Education, 2014, vol.12 (3), pp. 523-527.

[2] Li Peixian, Application of MATLB in teaching of deformation monitoring data processing, Science of Surveying and Mapping, 2016, vol.41 (3), pp. 185-190. 
[3] Zha Jianfeng, Zhang Haojie, Zhao Jun and Wu Chenghong, Experiment teaching system of mining subsidence prediction based on MATLAB, China Mining Magazine, 2016, vol.25 (6), pp. 164-168.

[4] Gao Chao, Program implemention and optimization of surface subsidence prediction parameters determination based on Matlab curves fitting, Coal Mining Technology, 2018, vol.23 (1), pp. 33-37.

[5] Gao Chao, Program implemention and optimization of surface subsidence prediction parameters determination based on Matlab curves fitting, Coal Mining Technology, 2018, vol.23 (1), pp. 33-37.

[6] Luo Mingfeng, Matlab-realized visual $A^{*}$ path planning teaching platform, International Journal of Emerging Technologies in Learning, 2018, vol. 13(10), pp. 196-207. https://doi.org/10.3991/ijet.v13i10.9463

[7] H. Xu. B. Liu, Z Fang, New grey prediction model and its application in forecasting land subsidence in coal mine, Natural Hazards, 2014, vol.71 (2), pp. 1181-1194. https://doi.org/10.1007/s11069-013-0656-4

[8] Tao Qiuxiang, Zhai Min, The design and implementation of teaching for GPS network adjustment based on matlab, Geomatic \& Spatial Information Technology, 2016, vol.39 (3), pp. 9-12.

[9] Li Wei, Xu Aigong, Zhao Liang. Research on the conversion of coordinate measuring system based on matlab, Journal of China Coal Society, 2014, vol.39 (S1), pp. 88-92.

[10] Gao Caiyun, Cui Xi ming. Nonlinear time series of deformation forecasting using improved BP neural networks, Computer Modelling and New Technologies, 2014, vol.18 (8), pp. 249-253.

[11] Chen Lin, Huang Teng, Zheng Hao, The model improvement of wavelet neural network in deformation prediction, Science of Surveying and Mapping, 2017, vol.42 (9), pp. 112-115.

[12] Gao ning, Gao Caiyun, Cui Ximin, Grey envelope detection method for multidimensional gross error of deformation data, Journal of Geodesy and Geodynamics, 2012, vol.32 (6), pp. 99-102.

[13] Li Lianfang, Zhang Qiang, Program design for deformation monitoring network quasi stable adjustment, Technology and Economy in Areas of Communications, 2015, vol.17 (2), pp. 118-120.

[14] Stojanovic B, Milivojevic M, Ivanovic M, Adaptive system for dam behavior modeling based on linear regression and genetic algorithms ,Advances in Engineering Software,2013, vol.65 (10), pp. 182-190. https://doi.org/10.1016/j.advengsoft.2013.06.019

[15] Wang Jianmin, Zhang Jin, Deformation intelligent prediction model based on Gaussian process regression and application, Geomatics and Science of Wuhan University, 2018, vol.43 (2), pp. 248-253.

[16] Hu Chuanfu, Application of e-learning assessment based on AHP-BP algorithm in the cloud computing teaching platform, International Journal of Emerging Technologies in Learning, 2016, vol. 11(08), pp. 27-32. https://doi.org/10.3991/ijet.v11i08.6039

[17] Gao Caiyun, Wu Qiutang, Design and practice of surveying experiment system based on a virtual platform, International Journal of Emerging Technologies in Learning, 2017, vol. 12(04), pp. 53-61. https://doi.org/10.3991/ijet.v12i04.6924 
Paper-Visualization Teaching of Deformation Monitoring and Data Processing based on MATLAB

\section{$5 \quad$ Author}

Ning Gao is an associate professor in School of Geomatics \& Urban Spatial Informatics, Henan University of Urban Construction, Pingdingshan 467036, China (gaoninghaoyun@163.com).

Article submitted 09 November 2018. Final acceptance 10 December 2018. Final version published as submitted by the author. 